\title{
Testing the predicted mass-loss bi-stability jump at radio wavelengths
}

\author{
P. Benagliaa ${ }^{1,2}$, J. S. Vink ${ }^{3,4}$, J. Martí ${ }^{5}$, J. Maíz Apellániz ${ }^{6,7}$, B. Koribalski ${ }^{8}$, and P. A. Crowther ${ }^{9}$ \\ 1 Instituto Argentino de Radioastronomía, C.C.5, (1894) Villa Elisa, Argentina \\ ${ }^{2}$ Facultad de Cs. Astronómicas y Geofísicas, UNLP, Paseo del Bosque s/n, (1900) La Plata, Argentina \\ e-mail: pbenaglia@fcaglp.unlp.edu.ar \\ 3 Keele University, Astrophysics, Lennard-Jones Lab, ST5 5BG, UK \\ e-mail: jsv@astro.keele.ac.uk \\ 4 Imperial College, Blackett Laboratory, Prince Consort Road, London, SW7 2AZ, UK \\ 5 Departamento de Física, EPS, Universidad de Jaén, Campus Las Lagunillas s/n, Edif. A3, 23071 Jaén, Spain \\ e-mail: jmarti@ujaen.es \\ ${ }^{6}$ Instituto de Astrofísica de Andalucía, Camino bajo de Huétor 50, Granada 18008, Spain \\ e-mail: jmaiz@iaa.es \\ 7 Ramón y Cajal fellow, Ministerio de Educación y Ciencia, Spain \\ 8 Australia Telescope National Facility, CSIRO, PO Box 76, Epping, NSW 1710, Australia \\ e-mail: Baerbel.Koribalski@csiro.au \\ 9 Department of Physics and Astronomy, University of Sheffield, Hicks Building, Hounsfield Road, Sheffield S3 7RH, UK \\ e-mail: Paul.Crowther@sheffield.ac.uk
}

Received 19 January 2007 / Accepted 21 March 2007

\section{ABSTRACT}

\begin{abstract}
Context. Massive stars play a dominant role in the Universe, but one of the main drivers for their evolution, their mass loss, remains poorly understood.

Aims. In this study, we test the theoretically predicted mass-loss behaviour as a function of stellar effective temperature across the so-called "bi-stability" jump.

Methods. We observe OB supergiants in the spectral range O8-B3 at radio wavelengths to measure their thermal radio flux densities, and complement these measurements with data from the literature. We derive the radio mass-loss rates and wind efficiencies, and compare our results with $\mathrm{H} \alpha$ mass-loss rates and predictions based on radiation-driven wind models.

Results. The wind efficiency shows the possible presence of a local maximum around an effective temperature of $21000 \mathrm{~K}-$ in qualitative agreement with predictions. Furthermore, we find that the absolute values of the radio mass-loss rates show good agreement with empirical $\mathrm{H} \alpha$ rates derived assuming homogeneous winds - for the spectral range under consideration. However, the empirical mass-loss rates are larger (by a factor of a few) than the predicted rates from radiation-driven wind theory for objects above the bi-stability jump (BSJ) temperature, whilst they are smaller (by a factor of a few) for stars below the BSJ temperature. The reason for these discrepancies remains as yet unresolved. A new wind momenta-luminosity relation (WLR) for O8-B0 stars has been derived using the radio observations. The validity of the WLR as a function of the fitting parameter related to the force multiplier $\alpha_{\text {eff }}$ (Kudritzki \& Puls 2000) is discussed.

Conclusions. Our most interesting finding is that the qualitative behaviour of the empirical wind efficiencies with effective temperature is in line with the predicted behaviour, and this presents the first hint of empirical evidence for the predicted mass-loss bi-stability jump. However, a larger sample of stars around the BSJ needs to be observed to confirm this finding.
\end{abstract}

Key words. radio continuum: stars - stars: early-type - stars: mass-loss - stars: winds, outflows

\section{Introduction}

Massive stars are the main drivers for the evolution of galaxies: they are the prime contributors to the energy and momentum input into the interstellar medium through stellar winds and supernovae, they generate the bulk of ionising radiation, and they are important sources for the chemical enrichment of carbon, oxygen and nitrogen in the Universe. Their very evolution through subsequent stages ( $\mathrm{OB} \rightarrow \mathrm{LBV} \rightarrow \mathrm{WR} \rightarrow \mathrm{SN}$ ) is believed to be driven by mass loss (e.g. Chiosi \& Maeder 1986). Heger et al. (2003) have recently reviewed the way in which massive stars end their lives and they find that the type of remnant that is left - neutron star, black hole, or no remnant at all - is primarily dependent on the amount of mass lost via stellar winds.
The physical mechanism for these winds of massive OB stars has long been identified to be that of radiation pressure on millions of spectral lines (Lucy \& Solomon 1970; Castor et al. 1975: hereafter $\mathrm{CAK}$ ). These radiation-driven wind models predict the mass-loss rates $\dot{M}$ of O stars to depend strongly on the stellar luminosity, and the terminal flow velocity $\left(v_{\infty}\right)$ to be proportional to the escape velocity $\left(v_{\mathrm{esc}}\right)$, reaching wind velocities of thousands of $\mathrm{km} \mathrm{s}^{-1}$. Over the last decades, the theory has proven to be very successful in explaining the overall mass-loss properties of O stars, with $\dot{M}$ of O stars up to $10^{-5} M_{\odot} \mathrm{yr}^{-1}$ and a clear correlation between the wind terminal and the escape velocity: $\left(v_{\infty} / v_{\text {esc }}\right) \simeq 2.6$ (Kudritzki \& Puls 2000).

Nevertheless, there are many open issues in the field of massive star evolution and their winds - even during the "best 


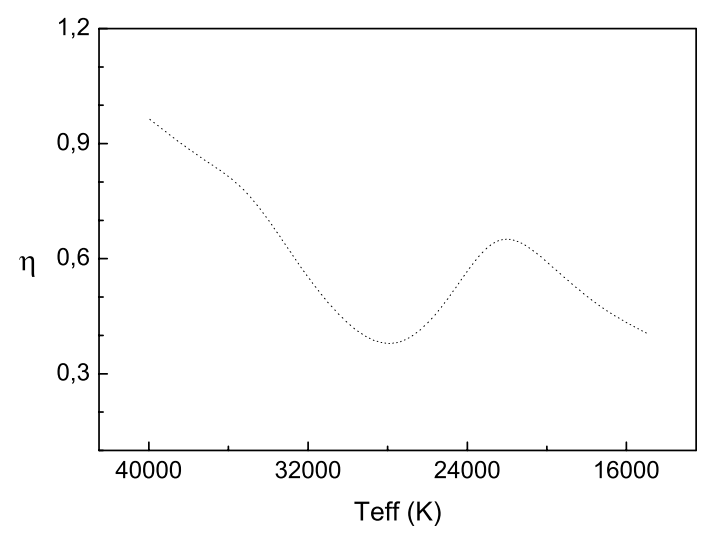

Fig. 1. The predicted wind efficiency number: $\eta=\left(\dot{M} v_{\infty}\right) /\left(L_{*} / c\right)$ as a function of effective temperature (from Vink et al. 2000a). Note the presence of a local maximum at the position of the bi-stability jump around $T_{\text {eff }}=21000 \mathrm{~K}$.

understood" O \& B evolutionary phases. One of them is that of wind clumping (e.g. Eversberg et al. 1998). Many modern evolution models (Maeder \& Meynet 2003; Limongi \& Chieffi 2006; Eldridge \& Vink 2006) use OB mass-loss rates from smooth wind Monte Carlo mass-loss predictions of Vink et al. (2000a,b). However, the sizes of these mass-loss rates have recently been questioned by a number of ultraviolet (UV) studies, and downward revisions by factors of five to ten have been suggested (e.g. Bouret et al. 2003; Fullerton et al. 2006). There are theoretical and observational indications (e.g. Evans et al. 2004) that the winds of $\mathrm{OB}$ stars are clumped, but what remains uncertain is by what factor the mass-loss rates could be over-predicted; is it as dramatic (i.e. factors $\sim 10$ ) as has been suggested by the UV studies, or is the clumping factor relatively small (a factor of $\sim$ two)?. It is relevant to note that a modest amount of wind clumping is actually required to match the Vink et al. (2000a, 2001) mass-loss predictions. If the clumping factor $(f)$ is only $\sim 5$ - the corresponding mass-loss rates are a factor $\sqrt{5}$ lower (see Repolust et al. 2004; Mokiem et al. 2007), massive star evolution is not anticipated to be dramatically affected by clumping. For a recent discussion on wind clumping from optical studies, we refer to Puls et al. (2006), and Davies et al. (2007).

Another topical issue is that of the "bi-stability jump" (BSJ): an empirical drop in the ratio $\left(v_{\infty} / v_{\text {esc }}\right)$ from 2.6 to 1.3 around temperatures of about $21000 \mathrm{~K}$ (Lamers et al. 1995). Theoretically, the wind characteristics as a function of stellar spectral type are best described in terms of the wind efficiency number: $\eta=\left(\dot{M} v_{\infty}\right) /\left(L_{*} / c\right)$, a measure for how much momentum from the photons is transferred to the ions of the outflowing wind. Vink et al. (2000a) computed a large grid of wind models as a function of effective temperature and presented the following overall behaviour of the mass-loss rate as a function of decreasing temperature (Fig. 1).

Over the temperature range between $50000 \mathrm{~K}$ down to $27500 \mathrm{~K}$, the mass-loss rates drop rapidly. The reason is that of a growing mismatch between the wavelengths of the maximum opacity in the UV and the flux maximum, which progressively moves from extreme UV (Ly continuum) to far UV, from early $\mathrm{O}$ to early B stars. The behaviour is however reversed around the BSJ, where $\eta$ is now predicted to increase by a factor of 2-3 to a local maximum when Fe IV recombines to the more efficient Fe III configuration (Vink et al. 1999; Vink 2000). Below the BSJ, the first effect returns, and $\eta$ is again predicted to decrease with temperature.
For B supergiants, Vink et al. (2000a) reported significant discrepancies between empirical and predicted mass-loss rates. Recent studies involving $\mathrm{H} \alpha$ (Trundle \& Lennon 2005; Crowther et al. 2006) and UV analyses (Prinja et al. 2005) have reconfirmed these findings. However, discrepancies between empirical UV rates and radiation-driven wind models have now also been reported for O stars (see Fullerton et al. 2006). It is currently unclear whether the reported discrepancies for B supergiants are due to the assumption of smooth winds (and the neglect of wind clumping) in the radiation-driven wind model for all spectral types, or instead that they are somehow related to the physics of the BSJ.

\subsection{The bi-stability jump}

The bi-stability jump was first discussed by Pauldrach \& Puls (1990) in the context of their model calculations of an individual star, the Luminous Blue Variable (LBV) P Cygni. The first empirical work on the BSJ for a sample of stars was that by Lamers et al. (1995), who found a discontinuity in the ratio $\left(v_{\infty} / v_{\mathrm{esc}}\right)$ at spectral type B1 $\left(T_{\text {eff }} \sim 21000 \mathrm{~K}\right)$. For the earlier-type stars the ratio is $\simeq 2.6$, but for the lower temperatures it drops to $\simeq 1.3$. They also discussed the possible existence of a second BSJ at spectral type A0. We note that Crowther et al. (2006) performed a sophisticated analysis using line-blanketed model atmospheres (of Hillier \& Millier 1998) of the temperatures of objects around the B1 BSJ and concluded the empirical BSJ in wind velocity represents a more gradual decrease than suggested by the step function of Lamers et al. (1995).

On the theoretical front, Vink et al. (1999) studied the nature of the winds around the BSJ and found a gradual change in wind properties, while they attributed the origin of the BSJ to a gradual change in the ionization of the Fe lines that drive the wind. For a range of stellar parameters and adopted velocity laws, Vink et al. $(1999,2000$ a) found an increase in the wind efficiency $\eta$ by a factor 2-3 independent of the assumed terminal velocity. When accounting for the empirical drop in terminal velocity, they found a jump in the mass-loss rate by a factor of five - for stars at the same luminosity. They argued that the jump in mass loss is accompanied by a decrease of the ratio $\left(v_{\infty} / v_{\text {esc }}\right)$, which is the observed bi-stability jump in terminal velocity. Using selfconsistent models they obtained ratios within 10 percent of the observed values around the jump (Vink et al. 1999, 2000b; Vink 2000). After a detailed investigation of the line acceleration for models around the jump, they demonstrated that $\dot{M}$ increases at the BSJ due to an increase in the line acceleration of Fe III near the stellar photosphere.

The bi-stability mechanism may be the crucial piece of physics for understanding the least understood phases of massive star evolution, i.e. the LBV and the B[e] phases. Vink \& de Koter (2000) found that the bi-stability jump may explain the mass-loss variability of LBVs. Smith et al. (2004) showed that, under certain conditions (when stars have lost a lot of mass due to mass loss), the bi-stability jump may be able to explain the formation of pseudo-photospheres and possibly even the existence of Yellow Hypergiants. Kotak \& Vink (2006) recently suggested that the quasi-sinusoidal modulations in the radio lightcurves of a number of supernovae may be caused by variable LBV wind strengths, suggesting that LBVs may be the progenitors of some supernovae, which would have profound consequences for our most basic understanding of massive star evolution.

For rapidly rotating stars with radiative envelopes, the pole may be expected to be hotter than the stellar equator, as due to the Von Zeipel gravity darkening effect, implying that the BSJ 
could provide a higher mass loss from the equator than from the pole for B stars. This will give rise to a high density "diskforming region" at the stellar equator - possibly causing the B[e] phenomenon (Lamers \& Pauldrach 1991; Pelupessy et al. 2000; Cure et al. 2005).

However attractive the physics of the BSJ may be, the expected jump in mass loss has yet to be investigated on observational grounds, which is the prime goal of this study. We perform a comprehensive radio analysis of massive stars over the spectral range $\mathrm{O} 8 \mathrm{-B} 3$, corresponding to $T_{\text {eff }}$ in the critical range of $35000-15000 \mathrm{~K}$.

The contents of the paper is as follows. Section 2 briefly reviews the derivation of stellar mass-loss rates by means of radio data. Section 3 describes the stellar sample, including the radio observations carried out specifically for this work. In Sect. 4 we explain the adopted stellar parameters and the derivation of radio mass-loss rates. Section 5 presents the wind efficiencies and a wind momentum-luminosity relation obtained with radio data. In Sect. 6, we compare our radio results with theoretical predictions. In Sect. 7 we analyze the correlation between radio- and $\mathrm{H} \alpha$-derived mass-loss rates. Section 8 closes with a summary and the conclusions of the present study.

\section{Stellar mass-loss rates from radio observations}

The measurement of stellar mass-loss rates can be achieved by means of at least three methods, which involve observations at radio, optical $(\mathrm{H} \alpha)$, and UV wavelengths. Each technique retrieves information from a different part of the wind. Although the radio method is not as sensitive as the $\mathrm{H} \alpha$ or UV methods, the radio flux density method is generally accepted to be the most accurate (Abbott et al. 1980; Lamers \& Leitherer 1993). The reason is that the method does not require a priori knowledge of the excitation, ionization or velocity structure in the winds, as the other approaches do to a more or lesser extent.

Although the radio method is considered to be the most accurate, it nonetheless depends on the assumption that the radio flux density is predominantly thermal in nature. This may be quite a good assumption for the objects under consideration in this study, as we expect that non-thermal emission at these spectral types should not be important (even irrespective of the wavelength): all (13) targets in the range O8-B3 detected at two frequencies by Bieging et al. (1989), and by Scuderi et al. (1998) showed thermal spectral indices. Other potential inaccuracies in the radio mass-loss rates are due to uncertainties in the stellar distance $d$ and only slightly on the value of the terminal velocity of the winds. We discuss the effect of wind clumping in the last sections.

Despite the weakness of the thermal radio emission from OB stellar winds (usually less of $1 \mathrm{mJy}$ ), the number of closeby $(\leq 3 \mathrm{kpc})$ targets is enough to perform a statistical study. The thermal radio flux density $S_{v}$ at a frequency $v$, from an optically thick stellar wind, is related to the stellar mass-loss rate $\dot{M}$ as (Wright \& Barlow 1975; Panagia \& Felli 1975):

$\dot{M}=5.32 \times 10^{-4} \frac{\left(S_{v}\right)^{3 / 4} d^{3 / 2} v_{\infty} \mu}{Z \sqrt{\gamma g_{v} v}} \quad M_{\odot} \mathrm{yr}^{-1}$.

Here $\mu$ is the mean molecular weight of the ions, $Z$ the rms ionic charge, $\gamma$ the mean number of electrons per ion, and $g_{v}$ the Gaunt factor, approximated by:

$g_{v}=9.77\left[1+0.13\left(\log \left(0.4 T_{\mathrm{eff}}\right)^{3 / 2}(Z v)^{-1}\right)\right]$.

\section{Observations at radio wavelengths}

In order to derive mass-loss rates from continuum radio observations, for stars with effective temperatures around $21000 \mathrm{~K}$, we aimed at supergiants of spectral types in the range $\mathrm{O} 8$ to B3. We present here the results of our first observing campaign, with targets of spectral type spread over the mentioned range. The sample was completed with the addition of the results from previous observations taken from the literature and carried out by several authors.

The observing campaign consisted of 12 targets with declinations between $-57^{\circ}$ to $+37^{\circ}$. Eight of them were observed with the $\mathrm{NRAO}^{1}$ Very Large Array (VLA), while the southern ones were observed using the Australia Telescope Compact Array (ATCA) ${ }^{2}$. The stars can be identified in Table 1, with the acronyms ATCA or VLA (third column). The targets were required to be closeby and have as-accurate-as-possible determinations of terminal velocities. The integration time was estimated as the time needed to detect the radio flux density corresponding to the star mass-loss rate as predicted by Vink et al. recipe ${ }^{3}$ (Vink 2000).

\subsection{ATCA data}

The ATCA observations were conducted on June 26-27, 2005, at $6 \mathrm{~B}$ array configuration. The observing wavelengths were chosen at the $12 \mathrm{~mm}$ band, i.e. high frequencies, where not only the thermal flux density is stronger, but also the contribution of non-thermal emission to the radio flux density may be disregarded. The angular resolution is about $1^{\prime \prime}$. The observing frequencies were 17.728 and $17.856 \mathrm{GHz}$, and the total bandwidth was $128 \mathrm{MHz}$ in each of the two IFs. Due to bad weather $25 \%$ of the data were not useful, and had to be flagged. The sources were observed during 6 min scans, interleaved with 2 min scans of phase calibrator observations. The bandpass calibrator was 1253-055, and the flux density calibrator was PKS 1934-638 $\left(S_{12 \mathrm{~mm}}=1.03 \mathrm{Jy}\right)$ during both runs. Table 1 lists the phase calibrator and the integration time for each target source.

The data reduction and analysis were performed with the MIRIAD package. One star was detected, HD 148379, with a flux density of $0.28 \pm 0.05 \mathrm{mJy}$ (see Fig. 2). The synthesized beam for each source is given in Table 1. Table 2 shows the flux densities and 1- $\sigma$ errors, and, for the case of non-detections, the flux density value quoted as an upper limit corresponds to a 3- $\sigma$ value.

\subsection{VLA data}

The VLA targets were observed on September 9 and 17, 2005, at $\mathrm{C}$ array, during a total observing time of $8 \mathrm{~h}$. The flux density calibrator was $1331+305\left(S_{3.5 \mathrm{~cm}}=5.23 \mathrm{Jy}\right)$. The integration time on source, synthesized beam, and phase calibrator corresponding to each source are given in Table 1. The observations were carried out at $3.5 \mathrm{~cm}$ with two IF pairs of $50 \mathrm{MHz}$ bandwidth, to obtain a reasonable angular resolution with a moderate amount of calibration time, and lower noise receivers.

1 The National Radio Astronomy Observatory is a facility of the National Science Foundation operated under cooperative agreement by Associated Universities, Inc.

2 The Australia Telescope Compact Array is funded by the Commonwealth of Australia for operation as a National Facility by CSIRO.

${ }^{3}$ http://www .arm.ac.uk/ jsv/ 

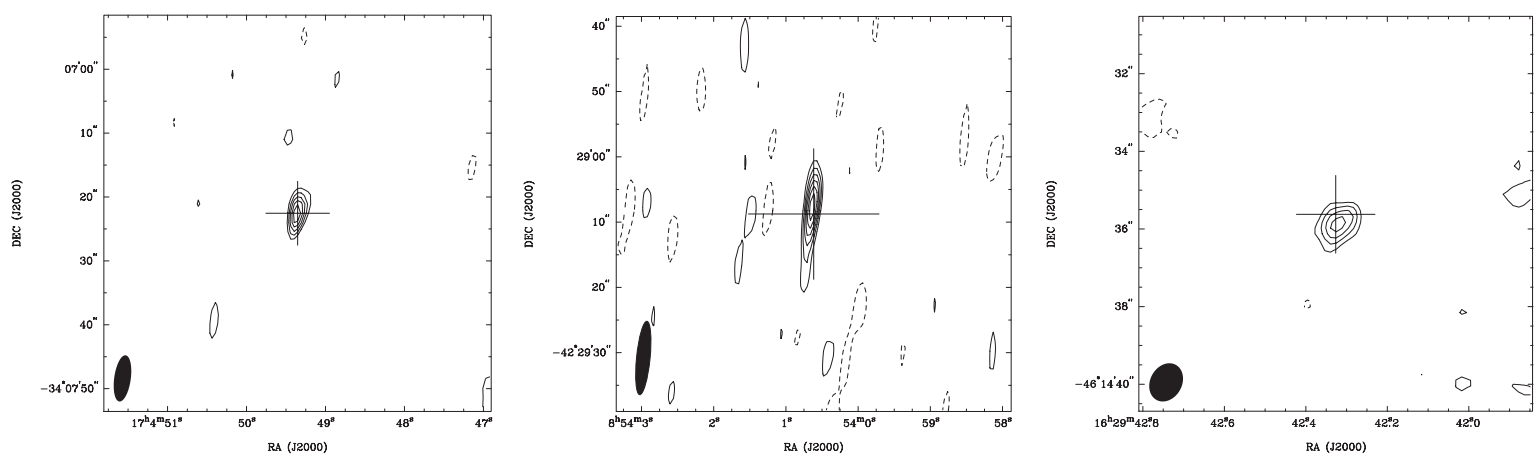

Fig. 2. Left panel: VLA radio continuum image of HD 154090 at $8.46 \mathrm{GHz}$. Contour levels: $-0.08,0.08(2 \sigma), 0.12,0.15,0.18$. and $0.21 \mathrm{mJy}$ beam $^{-1}$. Central panel: VLA radio continuum image of HD 76341 at $8.46 \mathrm{GHz}$. Contour levels: $-0.15,0.15(3 \sigma), 0.2,0.25,0.3$ and $0.35 \mathrm{mJy}$ beam $^{-1}$. Right panel: ATCA radio continuum image of HD 148379 at $17.8 \mathrm{GHz}$. Contour levels: $-0.1,0.15(3 \sigma), 0.2,0.25$, and $0.3 \mathrm{mJy} \mathrm{beam}^{-1}$. The optical position of the star is marked with a cross, and the synthesized beam is displayed in the bottom left corner of each image.

Table 1. Observations details.

\begin{tabular}{llrrr}
\hline \hline Star & $\begin{array}{l}\text { Phase } \\
\text { Cal. }\end{array}$ & Tel. & $\begin{array}{r}\text { Int. } \\
\text { time } \\
(\mathrm{h})\end{array}$ & $\begin{array}{r}\text { Synth. } \\
\text { beam } \\
\text { (arcsec) }\end{array}$ \\
\hline HD 42087 & $0559+238$ & VLA & 0.81 & $2.2 \times 1.8$ \\
HD 43384 & $0559+238$ & VLA & 0.83 & $2.2 \times 1.8$ \\
HD 47432 & $0641-033$ & VLA & 0.75 & $2.5 \times 2.3$ \\
HD 76341 & $0828-375$ & VLA & 0.50 & $11.3 \times 2.3$ \\
HD 112244 & j1326-5256 & ATCA & 3.18 & $0.8 \times 0.4$ \\
HD 148379 & $1600-44$ & ATCA & 3.58 & $1.0 \times 0.8$ \\
HD 154090 & $1626-298$ & VLA & 0.75 & $7.3 \times 2.6$ \\
HD 156154 & $1744-312$ & VLA & 0.5 & $7.8 \times 2.6$ \\
HD 157246 & $1657-56$ & ATCA & 6.9 & $0.6 \times 0.5$ \\
HD 165024 & $1740-517$ & ATCA & 6.36 & $0.7 \times 0.5$ \\
HD 204172 & $2052+365$ & VLA & 1.25 & $3.0 \times 2.7$ \\
BD-11 4586 & $1832-105$ & VLA & 0.5 & $3.8 \times 2.7$ \\
\hline
\end{tabular}

The data was reduced and analyzed with the AIPS and MIRIAD routines. Two stars were detected above the noise: HD 76341, and HD 154090, with signal-to-noise ratio $\geq 6$ (see Fig. 2). The stellar field of HD 156154 showed weak emission at a 3- $\sigma$ level, and a 2- $\sigma$ (probable) noise source coincident with the stellar position. Diffuse emission over the field of HD 42087 precluded the detection of the stellar wind, also superposed to a 2MASS source, and the infrared source IRAS 06066+2307.

An extended source was discovered in the stellar field of BD-114586, centered at (RA, Dec) $)_{\mathrm{J} 2000}=$ $\left(18^{\mathrm{h}} 18^{\mathrm{m}} 6.744^{\mathrm{s}},-11^{\circ} 18^{\prime} 16.83^{\prime \prime}\right)$, with a total flux density of $2.8 \mathrm{mJy}$. No correlation at any wavelength was found for this source in the literature.

\subsection{Previous radio observations}

We searched the literature for continuum radio observations of late OB supergiants with spectral types between $\mathrm{O} 8$ to B3. Among the contributions considered, the comprehensive study by Bieging et al. (1989) on OB stars listed high-resolution radio observations of 16 such supergiants, six of which were detected. Lamers \& Leitherer (1993) presented seven radio detections. Scuderi et al. (1998) obtained radio flux densities - or upper limits - for ten candidates. We have also included isolated results from Stevens (2006) (private communication), Benaglia et al. (2001), and the latest radio flux densities detected by Puls et al. (2006) on two O9.5 targets. From the older studies (Bieging et al. 1989; Lamers \& Leitherer 1993), only detections were included, as the observations were carried out using the former
VLA less sensitive receivers. The star HD 193237 (observations by Scuderi et al. 1998) is to be discussed elsewhere amongst LBVs. The star HD 152408 is not included within the present sample due to its ambiguous classification as either "O8 Iafpe" or "WN9 ha" (see Bohannan \& Crowther 1999).

The star HD 149404 is listed by Lamers \& Leitherer (1993). It has been studied by Rauw et al. (2001), and Thaller et al. (2001), through optical spectroscopy. They found evidences of a binary system, formed by an O7.5 I(f) and an ON9.7 I, with a period of $\sim 9 \mathrm{~d}$. Most of the radio emission would be produced in a colliding wind region; the results are consistent with a picture where the secondary star is undergoing Roche lobe overflow. Within this scenario, the radio flux density measured should come from the region of colliding winds, as well as the winds of both components. The mass-loss rate derived from the radio flux density is thus only an upper limit and we disregard from our upcoming analysis this object as well.

\subsection{Considerations on radio-detectability}

The O8 to B3 supergiant sample comprises 19 detections (up to $3 \mathrm{mJy}$ ) plus upper limits to 11 sources (typically below $0.3 \mathrm{mJy}$ ). With the aim of comparing the different radio flux densities measured towards the sample stars, we have extrapolated the values of $S_{v}$ to a unique frequency of $14.95 \mathrm{GHz}$, assuming that the thermal flux behaves as $S_{v} \propto v^{0.7}$ (Setia Gunawan et al. 2000; Williams 1996). In Fig. 3 we have plotted the quantity $S_{v} *(d / 1 \mathrm{kpc})^{2} \propto L$ as a function of the stellar effective temperature. We found no correlation of the corrected flux with stellar effective temperature, although the errors in stellar distances are strongly affecting the result.

No correlations between radio flux density and terminal velocity, distance or visual magnitude were found, as expected.

\section{Stellar parameters and mass-loss rates}

Table 2 lists the 30 selected stars in two groups (observed by us, and compiled from the literature), ordered by HD number, together with other observational stellar parameters.

The spectral types for O stars were taken from the GOS catalogue (Maíz Apellániz et al. 2004), Crowther et al. (2006), and Puls et al. (2006). The various references on spectral types of B stars can be seen in Table 2 . The $V, B$ and absolute adopted magnitudes are also given.

Most of the stellar wind terminal velocities quoted were published by Howarth et al. (1997). For four objects, the values 
Table 2. Supergiants with radio observations: measurements.

\begin{tabular}{|c|c|c|c|c|c|c|c|c|c|c|}
\hline Star & Sp. Class. & Observ. & $\overline{V, B}$ & $\begin{array}{r}v_{\mathrm{W}} \\
\left(\mathrm{km} \mathrm{s}^{-1}\right) \\
\end{array}$ & $M_{\mathrm{V}}$ & $\begin{array}{r}d \\
(\mathrm{kpc})\end{array}$ & $\begin{array}{r}e_{d} \\
(\mathrm{kpc})\end{array}$ & $\begin{array}{r}v \\
(\mathrm{GHz})\end{array}$ & $\begin{array}{r}S_{v} \\
(\mathrm{mJy})\end{array}$ & $\begin{array}{r}e_{S} \\
(\mathrm{mJy})\end{array}$ \\
\hline HD 42087 & $\mathrm{~B} 2.5 \mathrm{Ib}^{a}$ & this work & $5.76,5.96^{b}$ & $735^{c}$ & $-6.4^{d}$ & $1.4^{e}$ & 0.3 & 8.640 & $<0.14$ & \\
\hline HD 43384 & B3 $\mathrm{Iab}^{f}$ & this work & $6.26,6.71^{g}$ & $760^{c}$ & $-7.2^{d}$ & $1.4^{e}$ & 0.3 & 8.640 & $<0.24$ & \\
\hline HD 47432 & $09.7 \mathrm{Ib}^{h}$ & this work & $6.22,6.36^{i}$ & $1590^{c}$ & $-6.28^{j}$ & $1.7^{e}$ & 0.4 & 8.640 & $<0.15$ & \\
\hline HD 76341 & O9 $\mathrm{Ib}^{k}$ & this work & $7.16,7.46^{i}$ & $1520^{c}$ & $-6.29^{j}$ & $1.9^{e}$ & 0.4 & 8.640 & 0.38 & 0.05 \\
\hline HD 112244 & $\mathrm{O} 8.5 \operatorname{Iab}(\mathrm{f})^{l}$ & this work & $5.38,5.40^{i}$ & $1575^{c}$ & $-6.29^{j}$ & $1.5^{e}$ & 0.3 & 17.79 & $<0.3$ & \\
\hline HD 148379 & B1.5 Iape $^{m}$ & this work & $5.34,5.80^{n}$ & $510^{c}$ & $-7.5^{d}$ & $1.3^{e}$ & 0.3 & 17.79 & 0.28 & 0.05 \\
\hline HD 154090 & $\mathrm{~B} 0.7 \mathrm{Ia}^{d}$ & this work & $4.87,5.13^{d}$ & $915^{d}$ & $-6.8^{d}$ & $1.1^{d}$ & 0.3 & 8.640 & 0.23 & 0.04 \\
\hline HD 156154 & O8 Iab(f) ${ }^{l}$ & this work & $8.05,8.65^{i}$ & $1530^{\circ}$ & $-6.3^{j}$ & $2.2^{e}$ & 0.5 & 8.640 & $<0.15$ & \\
\hline HD 157246 & $\mathrm{~B} 1 \mathrm{Ib}^{m}$ & this work & $3.34,3.21^{b}$ & $735^{c}$ & $-6.8^{d}$ & $1.1^{e}$ & 0.2 & 17.79 & $<0.18$ & \\
\hline HD 165024 & $\mathrm{~B} 2 \mathrm{Ib}^{m}$ & this work & $3.66,3.58^{b}$ & $1185^{c}$ & $-7.2^{d}$ & $0.8^{e}$ & 0.2 & 17.79 & $<0.18$ & \\
\hline HD 204172 & $\mathrm{~B} 0 \mathrm{Ib}^{h}$ & this work & $5.94,5.84^{p}$ & $1685^{c}$ & $-6.4^{d}$ & $3.0^{e}$ & 0.7 & 8.640 & $<0.08$ & \\
\hline BD-11 4586 & $\mathrm{O} 8 \mathrm{Ib}(\mathrm{f})^{q}$ & this work & $9.4,10.4^{i}$ & $1530^{\circ}$ & $-6.3^{j}$ & $1.8^{e}$ & 0.4 & 8.640 & $<0.3$ & \\
\hline HD 2905 & $\mathrm{BC} 0.7 \mathrm{Ia}^{d}$ & SPS98 & $4.16,4.30^{d}$ & $1105^{d}$ & $-7.1^{d}$ & $1.1^{d}$ & 0.3 & 8.450 & 0.4 & 0.03 \\
\hline HD 30614 & $\mathrm{O} 9.5 \mathrm{Ia}^{d}$ & SPS98 & $4.29,4.33^{i}$ & $1560^{d}$ & $-6.6^{d}$ & $1.0^{d}$ & 0.2 & 14.95 & 0.65 & 0.13 \\
\hline HD 37128 & $\mathrm{~B} 0 \mathrm{Ia}^{d}$ & SPS98 & $1.70,1.51^{d}$ & $1910^{d}$ & $-6.3^{d}$ & $0.4^{d}$ & 0.1 & 14.95 & 1.4 & 0.1 \\
\hline HD 37742 & $09.7 \mathrm{Ib}^{r}$ & LL93 & $1.76,1.55^{i}$ & $1860^{c}$ & $-6.3^{j}$ & $0.4^{e}$ & 0.1 & 8.000 & 0.89 & 0.04 \\
\hline HD 41117 & $\mathrm{~B} 2 \mathrm{Ia}^{d}$ & SPS98 & $4.63,4.91^{d}$ & $510^{c}$ & $-7.6^{d}$ & $1.5^{d}$ & 0.4 & 14.95 & 0.63 & 0.13 \\
\hline HD 80077 & $\mathrm{~B} 2 \mathrm{Ia}+{ }^{s}$ & LCK95 & $7.56,8.90^{t}$ & $140^{t}$ & $-7.2^{t}$ & $3.0^{p}$ & 1.2 & 8.640 & 0.5 & 0.11 \\
\hline HD 151804 & O8 $\mathrm{Iaf}^{l}$ & LL93 & $5.22,5.26^{u}$ & $1445^{c}$ & $-6.3^{j}$ & $1.9^{v}$ & 0.3 & 4.800 & 0.4 & 0.1 \\
\hline HD 152236 & $\mathrm{~B} 1.5 \mathrm{Ia}+{ }^{d}$ & Ste06 & $4.73,5.21^{d}$ & $390^{d}$ & $-8.8^{d}$ & $2.0^{d}$ & 0.3 & 8.640 & 2.4 & 0.1 \\
\hline HD 152424 & OC9.7 $\mathrm{Ia}^{r}$ & LL93 & $6.31,6.71^{i}$ & $1760^{c}$ & $-6.3^{j}$ & $1.7^{e}$ & 0.4 & 8.000 & 0.21 & 0.04 \\
\hline HD 163181 & BN0.5 Iap & BCK01 & $6.60,7.15^{x}$ & $520^{c}$ & $-6.1^{d}$ & $1.6^{e}$ & 0.4 & 8.640 & 0.44 & 0.05 \\
\hline HD 169454 & $\mathrm{~B} 1 \mathrm{Ia}^{y}$ & BAC89 & $6.65,7.39^{x}$ & $850^{z}$ & $-6.8^{d}$ & $0.9^{e}$ & 0.2 & 15.00 & 1.9 & 0.1 \\
\hline HD 190603 & $\mathrm{~B} 1.5 \mathrm{Ia}+{ }^{d}$ & SPS98 & $5.65,6.19^{d}$ & $485^{d}$ & $-7.5^{d}$ & $1.5^{e}$ & 0.4 & 14.95 & $<0.7$ & \\
\hline HD 194279 & $\mathrm{~B} 2 \mathrm{Ia}^{d}$ & SPS98 & $7.05,8.07^{d}$ & $550^{d}$ & $-7.0^{d}$ & $1.2^{d}$ & 0.3 & 8.450 & 0.44 & 0.1 \\
\hline HD 195592 & $09.5 \mathrm{I}^{l}$ & SPS98 & $7.08,7.95^{i}$ & $1765^{\circ}$ & $-6.3^{j}$ & $1.0^{e}$ & 0.2 & 14.95 & 0.9 & 0.13 \\
\hline HD 198478 & $\mathrm{~B} 2.5 \mathrm{Ia}^{d}$ & SPS98 & $4.86,5.28^{d}$ & $470^{d}$ & $-6.4^{d}$ & $0.8^{d}$ & 0.2 & 8.450 & $<0.27$ & \\
\hline HD 209975 & $09.5 \mathrm{Ib}^{z}$ & PMS06 & $5.10,5.18^{i}$ & $2050^{z}$ & $-5.45^{z}$ & $0.8^{z}$ & 0.2 & 14.94 & 0.422 & 0.12 \\
\hline CygOB2-10 & O9.5 I & PMS06 & $9.8811 .47^{\alpha}$ & $1650^{z}$ & $-6.95^{z}$ & $1.7^{z}$ & 0.2 & 14.94 & 0.3 & 0.1 \\
\hline BD-14 5037 & $\mathrm{~B} 1.5 \mathrm{Ia}^{\beta}$ & SPS98 & $8.22,9.58^{x}$ & $750^{\circ}$ & $-7.4^{\circ}$ & $1.0^{e}$ & 0.2 & 14.95 & 1.1 & 0.2 \\
\hline
\end{tabular}

${ }^{a}$ Perryman et al. (1997); ${ }^{b}$ Johnson et al. (1966); ${ }^{c}$ Howarth et al. (1997); ${ }^{d}$ Crowther et al. (2006); ${ }^{e}$ derived with CHORIzos: see text; ${ }^{f}$ Lesh et al. (1968); ${ }^{g}$ Moffett \& Barnes (1979); ${ }^{h}$ Walborn (1976); ${ }^{i} \mathrm{GOS}$ Catalogue (Maíz Apellániz et al. 2004); ${ }^{j}$ Martins et al. (2005); ${ }^{k} \mathrm{Garrison}$ et al. (1977); ${ }^{l}$ Walborn (1973); ${ }^{m}$ Hiltner et al. (1969); ${ }^{n}$ Feinstein \& Marraco (1979); ${ }^{o}$ Prinja et al. (1990); ${ }^{p}$ Crawford et al. (1971); ${ }^{q}$ Walborn (1982); ${ }^{r}$ Walborn

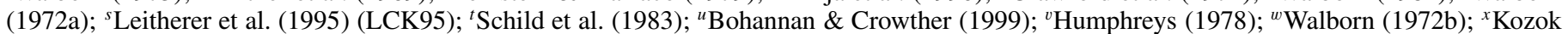
et al. (1985); ${ }^{y}$ Bieging et al. (1989) (BAC89); ${ }^{z}$ Puls et al. (2006) (PMS06); ${ }^{\alpha}$ Massey \& Johnson (1991); ${ }^{\beta}$ Morgan et al. (1955). SPS98: Scuderi et al. (1998). LL93: Lamers \& Leitherer (1993). Ste06: I.R. Stevens (private communication). BCK01: Benaglia et al. (2001).

were taken from the interpolations by Prinja et al. (1990), their Table 3, and involved relatively large errors. For the rest, we have adopted errors of $10 \%$.

To achieve the goal of a uniform database, we have established a common temperature scale for O8-B3 supergiants, rederived the stellar distances, and calculated the mass-loss rates for all stars in the sample using those consistent parameters.

The effective temperature scale was established taking into account the latest work by Crowther et al. (2006) for individual cases whenever possible, and also from interpolation using their Table 4. For some $\mathrm{O}$ supergiants the values were taken from the models by Martins et al. (2005). Isolated values are from Lamers \& Leitherer (1993) and Puls et al. (2006). The resulting scale is shown in Fig. 4. We have assigned to the thirteen different spectral types and subtypes covered, from 08 to B3, a correlated number (from 2 to 14). Figure 4 plots the stellar effective temperature as a function of this integer number. Spectral types were used to derive temperatures for the stars in the sample. The uncertainties introduced by the discrete nature of the spectral classification scheme, the current state of the art of hot-star atmosphere modeling, and the possible slight mis-classifications are expected to be of the order of $2000 \mathrm{~K}$ for late-O stars and
$1500 \mathrm{~K}$ for early-B stars. The effective temperature adopted for each star is listed in Table 3.

References for the adopted stellar luminosities are quoted in Table 3. The effective temperatures and luminosities of HD 42087, HD 148379, and HD 151804 have been derived specifically for this work by means of $\mathrm{H} \alpha$ profile fitting (see Sect. 7).

In order to derive the stellar masses, needed in turn to obtain predicted mass-loss rates (see Sect. 6), we used the calibration of surface gravity $(g)$ with effective temperature published by Crowther et al. (2006) (see their Fig. 1, which was built using SMC data presented by Trundle \& Lennon 2004). The likely range in the stellar mass for each star can be derived allowing a deviation by up to 0.2 dex in $\log (g)$.

Distances were derived using CHORIzos (Maíz Apellániz 2004) by comparing the observed with $B V J H K_{\mathrm{s}}$ photometry with low-gravity Kurucz atmospheres. The temperature for each star was taken from Table 3 and both $E_{4405-5495}$ and $R_{5495}$ (the monochromatic equivalents to $E_{B-V}$ and $R_{V}$, respectively, were left as free parameters). CHORIZOS is a Bayesian code that finds all the solutions for the general inverse photometric problem in which $N$ parameters (two in this case) are fitted to $M$ independent colors (three in this case). After applying CHORIzOs to 
Table 3. Adopted stellar parameters and results.

\begin{tabular}{|c|c|c|c|c|c|c|c|c|c|c|}
\hline Star & Sp. type & $\begin{array}{l}T_{\text {eff }} \\
(\mathrm{K})\end{array}$ & $\log \left(L_{*} / L_{\odot}\right)$ & $\log (g)$ & $\begin{array}{r}M_{*} \\
\left(M_{\odot}\right)\end{array}$ & $\eta$ & $\dot{M}$ & $\begin{array}{r}e_{\dot{M}} \\
0^{-6}\end{array}$ & $\underset{M_{\odot}}{\dot{M}_{\text {pred }}} \mathrm{yr}^{-1}$ & $\dot{M}_{\mathrm{H} \alpha}$ \\
\hline HD 156154 & O8 Iab(f) & $33179^{a}$ & $5.68^{a}$ & 3.10 & 19.2 & & $<4.4$ & & 3.2 & \\
\hline HD 151804 & O8 Iaf & $29000^{b}$ & $5.88^{b}$ & 3.00 & 41.4 & $1.0 \pm 0.12$ & 10.4 & 3.70 & 3.2 & $16.0^{b}$ \\
\hline BD-11 4586 & O8 Ib(f) & $33179^{a}$ & $5.68^{a}$ & 3.10 & 19.2 & & $<5.6$ & & 3.2 & $(2.6)^{c}$ \\
\hline HD 112244 & O8.5 Iab(f) & $32274^{a}$ & $5.65^{a}$ & 3.10 & 20.0 & & $<3.0$ & & 2.6 & \\
\hline HD 76341 & O9 Ib & $31368^{a}$ & $5.61^{a}$ & 3.05 & 18.2 & $1.3 \pm 0.17$ & 7.2 & 2.51 & 2.1 & \\
\hline CygOB2-10 & O9.5 I & $29700^{d}$ & $5.82^{d}$ & 3.00 & 32.8 & $0.5 \pm 0.07$ & 4.4 & 1.77 & 2.7 & $2.7^{d, e}$ \\
\hline HD 195592 & O9.5 I & $29000^{f}$ & $5.60^{f}$ & 3.00 & 21.7 & $1.0 \pm 0.14$ & 4.8 & 1.69 & 1.2 & $3.8^{d}$ \\
\hline HD 30614 & O9.5 Ia & $29000^{f}$ & $5.63^{f}$ & 3.00 & 23.3 & $0.6 \pm 0.08$ & 3.3 & 1.22 & 1.5 & $5.0^{f}$ \\
\hline HD 209975 & O9.5 Ib & $32000^{d}$ & $5.31^{d}$ & 3.10 & 9.5 & $1.2 \pm 0.15$ & 2.4 & 0.94 & 0.7 & $1.1^{d}$ \\
\hline HD 37742 & O9.7 Ib & $28500^{f}$ & $5.57^{a}$ & 2.95 & 19.4 & $0.4 \pm 0.05$ & 1.5 & 0.49 & 0.9 & $(1.8)^{g}$ \\
\hline HD 47432 & O9.7 Ib & $28500^{f}$ & $5.57^{a}$ & 2.96 & 19.8 & & $<3.1$ & & 1.1 & $(1.9)^{h}$ \\
\hline HD 152424 & OC9.7 Ia & $28500^{f}$ & $5.57^{a}$ & 2.96 & 19.8 & $1.0 \pm 0.14$ & 4.5 & 1.65 & 1.0 & \\
\hline HD 37128 & B0 Ia & $27000^{f}$ & $5.44^{f}$ & 2.90 & 15.9 & $0.5 \pm 0.07$ & 1.6 & 0.54 & 0.5 & $2.3^{f}$ \\
\hline HD 204172 & B0 Ib & $27500^{f}$ & $5.50^{f}$ & 2.93 & 18.2 & & $<5.0$ & & 0.7 & \\
\hline HD 163181 & BN0.5 Iap & $26000^{f}$ & $5.57^{a}$ & 2.85 & 22.4 & $0.1 \pm 0.02$ & 2.1 & 0.73 & 2.5 & \\
\hline HD 154090 & B0.7 Ia & $22500^{f}$ & $5.48^{f}$ & 2.60 & 18.1 & $0.2 \pm 0.03$ & 1.3 & 0.48 & 3.2 & $1.0^{f}$ \\
\hline HD 2905 & BC0.7 Ia & $21500^{f}$ & $5.52^{f}$ & 2.53 & 20.3 & $0.4 \pm 0.05$ & 2.5 & 0.84 & 2.3 & $2.0^{f}$ \\
\hline HD 169454 & B1 Ia & $21500^{f}$ & $5.45^{f}$ & 2.53 & 17.3 & $0.5 \pm 0.06$ & 3.4 & 1.14 & 2.9 & \\
\hline HD 157246 & B1 Ib & $20800^{i}$ & $5.45^{f}$ & 2.45 & 16.4 & & $<0.6$ & & 3.4 & \\
\hline BD-14 5037 & B $1.5 \mathrm{Ia}$ & $20500^{f}$ & $5.60^{f}$ & 2.45 & 24.5 & $0.2 \pm 0.04$ & 2.5 & 1.18 & 4.9 & $(3.4)^{j}$ \\
\hline HD 152236 & B1.5 Ia+ & $18000^{f}$ & $6.10^{f}$ & 2.20 & 73.4 & $0.1 \pm 0.02$ & 8.3 & 2.70 & 41.7 & $6^{f}$ \\
\hline HD 190603 & B1.5 Ia+ & $18500^{f}$ & $5.57^{f}$ & 2.25 & 21.8 & & $<2.1$ & & 6.8 & $2.5^{f}$ \\
\hline HD 148379 & B1.5 Iape & $18500^{b}$ & $5.56^{b}$ & 2.25 & 21.3 & $0.1 \pm 0.01$ & 0.8 & 0.29 & 5.8 & $1.5^{b}$ \\
\hline HD 194279 & B2 Ia & $19000^{f}$ & $5.37^{f}$ & 2.30 & 13.9 & $0.2 \pm 0.02$ & 1.5 & 0.58 & 3.3 & $1.1^{f}$ \\
\hline HD 41117 & B2 Ia & $19000^{f}$ & $5.65^{f}$ & 2.30 & 26.4 & $0.1 \pm 0.02$ & 2.1 & 0.76 & 8.5 & $1.0^{f}$ \\
\hline HD 80077 & B2 Ia+ & $18500^{f}$ & $5.40^{f}$ & 2.25 & 14.7 & $0.1 \pm 0.01$ & 1.7 & 0.69 & 30.2 & \\
\hline HD 165024 & B2 Ib & $18000^{f}$ & $5.40^{f}$ & 2.20 & 14.7 & & $<0.6$ & & 0.9 & \\
\hline HD 198478 & B2.5 Ia & $16500^{f}$ & $5.03^{f}$ & 2.05 & 6.3 & & $<0.5$ & & 1.1 & $0.2^{f}$ \\
\hline HD 42087 & B $2.5 \mathrm{Ib}$ & $19000^{b}$ & $5.08^{b}$ & 2.30 & 7.1 & & $<1.1$ & & 0.9 & $0.2^{b}$ \\
\hline HD 43384 & B3 Iab & $15500^{f}$ & $4.88^{h}$ & 1.95 & 4.4 & & $<1.7$ & & 0.3 & $(0.3)^{h}$ \\
\hline
\end{tabular}

${ }^{a}$ Martins et al. (2005); ${ }^{b}$ this work; ${ }^{c}$ Scuderi et al. (1992); ${ }^{d}$ Puls et al. (2006); ${ }^{e}$ Herrero et al. (2002); ${ }^{f}$ Crowther et al. (2006); ${ }^{g}$ Lamers \& Leitherer (1993); ${ }^{h}$ Morel et al. (2004); ${ }^{i}$ Lamers et al. (1995); ${ }^{j}$ Scuderi et al. (1998).

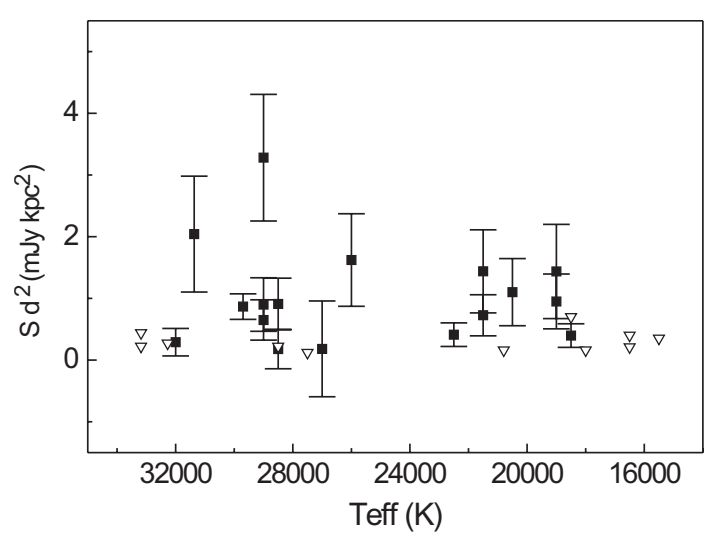

Fig. 3. Radio flux density corrected for the stellar distance, as a function of effective temperature. The squares represent detected stars, whilst the triangles represent radio flux density upper limits. The star HD 80077 was excluded due to its large error in distance $(\sim 40 \%)$.

obtain the unextinguished photometry, the spectroscopic distance was calculated using the values of $M_{V}$ in Table 2, assuming uncertainties between 0.5 and 1.0 mag depending on the spectral type. In all cases we found that the largest contribution to the final uncertainty in the distance, $e_{d}$, is that of the uncertainty in $M_{V}$ and not that of the photometry itself.

We investigated the membership of the sample stars to stellar clusters or OB associations. For those stars belonging

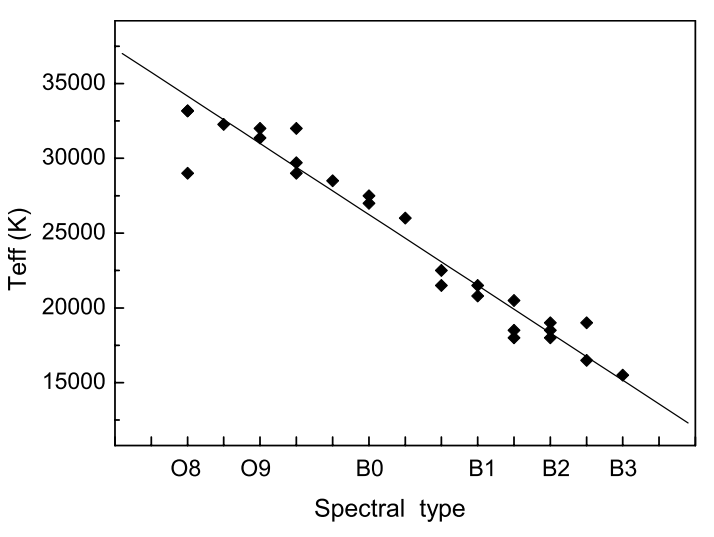

Fig. 4. The adopted stellar effective temperatures as a function of spectral type, for OB supergiants. The straight line represents the fit $y=$ $37327( \pm 387)-1583( \pm 44) x$, where $x$ is an index running on the different spectral types (from 2 to 14 ), and $y=T_{\text {eff }}$.

to stellar groups, we found that in most cases, our obtained individual distances agree well between errors, with those of the clusters/associations. For the star HD 151804, catalogued as a member of Sco OB1 (Humphreys 1978, $d=1.91 \mathrm{kpc}$ ), the association distance was adopted. Very recently, the distance to HD 169454 was established as $1.36 \mathrm{kpc}$ (Hunter et al. 2006). For the sake of consistency, we have adopted the distance derived here. 


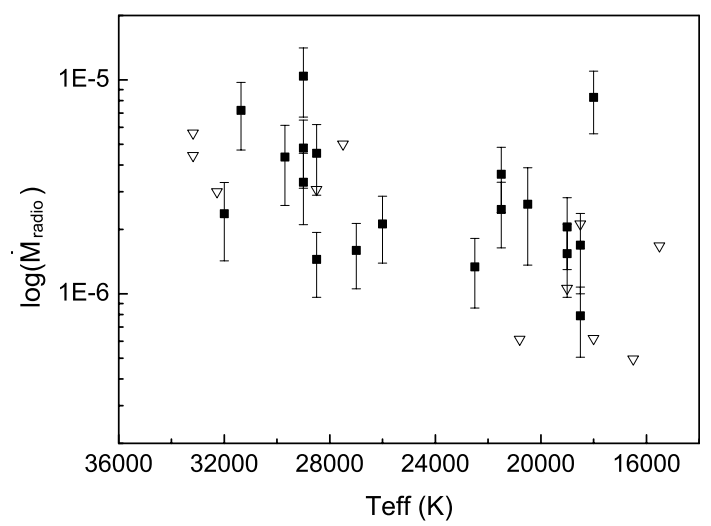

Fig. 5. Mass loss rates derived from radio continuum observations, as a function of effective temperature (squares), and upper limits (open triangles).

In order to derive the radio mass-loss rate values for previously observed stars, we have taken into account the radio flux density at the highest frequency whenever possible (see Table 2).

All mass-loss rates values were derived using Eq. (1), and assuming the measured flux density is thermal. This looks like a reasonable hypothesis, as all but one star (HD 151804) were observed at $v \geq 8 \mathrm{GHz}$. Besides, the sample stars that have been observed by Bieging et al. (1989) and Scuderi et al. (1998) at more than one frequency, have shown thermal emission. For all but one star we have adopted abundances of $Y_{\mathrm{H}}=0.85, Y_{\mathrm{He}}=0.15$, and assumed that at the temperatures considered, all $\mathrm{H}$ is ionized, while He is singly ionized. Consequently $\mu=1.45, Z=$ $\gamma=1$. The differences in the mass-loss rate results in considering $\mu=1.3-1.6$ were lower than $12 \%$. If the $\mathrm{He}$ is doubly ionized in $\mathrm{O}$ stars, the mass-loss rates derived here are underestimated by about $30 \%$, although we find this unlikely given the stellar effective temperatures involved. For HD 151804, we adopted $Y_{\mathrm{He}}=0.25$. Errors in $\mu, Z$, and $\gamma$ were taken as $10 \%$; $\sigma_{L}=0.5 L_{*}$. Mass-loss rate values and the corresponding errors derived from error propagation in Eq. (1) are listed in Table 3, and plotted in Fig. 5. In the case of the non-detections, a 3- $\sigma$ flux density upper limit was used, and the corresponding massloss rates are upper limits.

\section{Wind efficiencies}

In order to better compare radio results with results from other wavelengths or models, we proceeded to compute the wind efficiencies corresponding to the radio mass-loss rates. The values are shown in Table 3, and in Fig. 6 with filled squares. At $T_{\text {eff }} \approx 21.5 \mathrm{kK}$ there are two (squares) stars with wind efficiencies well above those of their neighbours: HD 169454 and HD 2905. The star HD 169454 has been represented twice, for wind efficiencies derived according to two possible distances (see Sect. 4): with a square when the distance adopted here is used, and with a triangle when the Hunter et al. (2006) value is used.

We wish to check if the empirical shapes of the wind efficiency could be an artifact of the fact that we observe objects with different masses and luminosities. This necessarily introduces a scatter in $\eta$, but we may be able to correct for this. The correction was derived in the following manner. From models for different parameter sets of $\left(M_{*}, L_{*}\right)$, we obtain the corresponding wind efficiencies. We choose a parameter set for which $\eta=1$ for the maximum effective temperature and $v_{\infty} / v_{\text {esc }}$ ratio (see Fig. 6

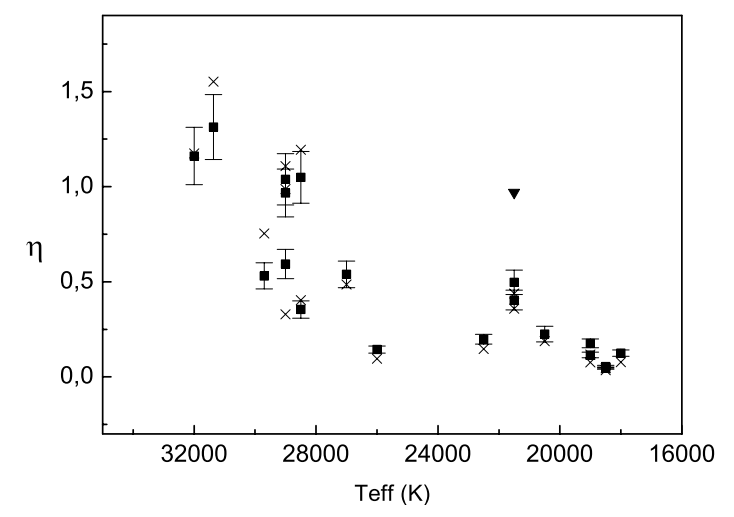

Fig. 6. Observed wind efficiency versus effective temperature (squares). Crosses represent "corrected" values (see text). The triangle corresponds to the star HD 169454 if the Hunter et al. (2006) distance is considered. Note the possible presence of a local maximum at the predicted position of the BSJ.

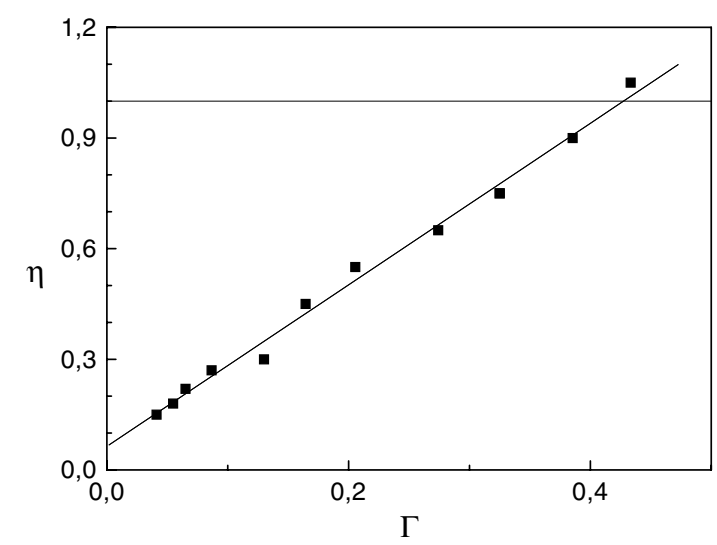

Fig. 7. Wind efficiencies taken for the twelve models from Fig. 5 from Vink et al. (2000a), as a function of the Eddington factor $\Gamma$. The straight line represents unity.

from Vink et al. 2000a). The $\eta$ values were plotted as a function of their Eddington factor $\left(\propto M_{*} / L_{*}\right)$, resulting in Eddingtoncorrected $\eta$ values (Fig. 7). For each target star we can compute the $\eta$ correction value according to its Eddington factor, and divide the uncorrected $\eta$ by the correction value. The results are also shown in Fig. 6. Though an additional source of scatter is being introduced as a result of this via the errors in stellar masses (not shown in the figure), the "corrected" wind efficiencies are similar to the original values. Consequently, hereafter we will use non-corrected values.

\subsection{The empirical wind-momentum relation}

The radio observations allowed us to estimate the mass-loss rates for the 19 detected stars in a uniform way. We have utilised this database to provide a radio derived calibration for the coefficients $D_{0}, x$ of the wind momenta-luminosity relation (WLR; see Kudritzki \& Puls 2000) for late O (O8-O9) and B0 supergiants. Our WLR is shown in Fig. 8. If the wind momenta equal $D=\dot{M}_{*} v_{\infty}\left(R / R_{\odot}\right)^{1 / 2}$, and $\log (D)=D_{0}+x \log \left(L / L_{\odot}\right)$, a linear fit yields the values $D_{0}=26.6 \pm 2.1 ; x=1.0 \pm 0.4$ when only the O stars (O8-O9) are included, and $D_{0}=25.0 \pm 2.7 ; x=1.3 \pm 0.5$ when the B0 stars are also included in the fit. For obvious reasons B1 stars are excluded as they are located at the BSJ, whilst for spectral types later than B1, we have too few datapoints to derive a reliable WLR. 


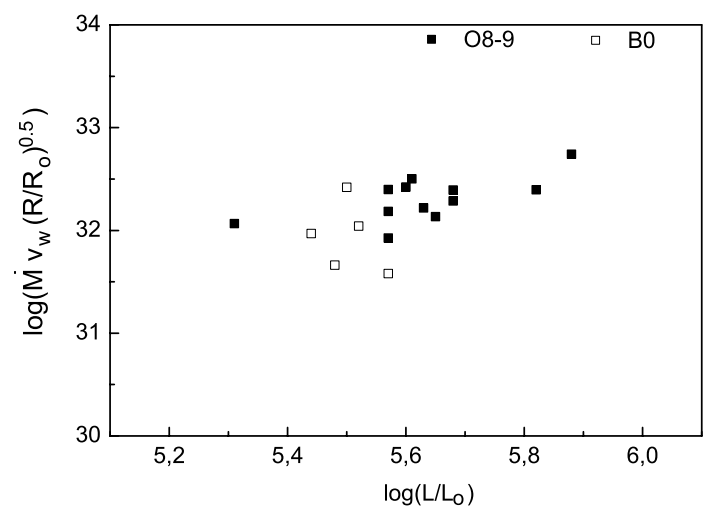

Fig. 8. Wind momenta $D$ of galactic late O-, and early B supergiants as a function of luminosity, derived using radio continuum observations.

We note the shallow slope of our derived WLR. For earlytype $\mathrm{O}$ stars the slope, $x$, is significantly larger. In standard radiation-driven wind theory, the slope, $x$, is the reciprocal value of the "effective" CAK force multiplier $\alpha_{\text {eff }}$ (see Kudritzki \& Puls for definitions). For early O-stars, this value is approximately $2 / 3$, the reason for which the stellar mass cancels and the WLR can be used in the first place.

Here, we find an empirical $\alpha_{\text {eff }}$ in the range $0.78-1$ for O8B0 stars, which may be consistent with the high $\alpha$ value of $\alpha^{\mathrm{MC}}=0.85$ predicted through Monte Carlo simulations by Vink et al. (1999) for models at $T_{\text {eff }}=30000 \mathrm{~K}$. A high value of $\alpha_{\text {eff }}$ may suggest a relatively large value for the ratio of $\left(v_{\infty} / v_{\mathrm{esc}}\right)$. Interestingly objects in this temperature range indeed show the largest $\left(v_{\infty} / v_{\text {esc }}\right)$ ratio according to Fig. 6 of Kudritzki \& Puls (2000). We note however that for these large values of $\alpha_{\text {eff }}$ (when $\alpha_{\text {eff }}$ no longer equals $2 / 3$ ), the concept of the WLR may lose its meaning. Investigations of the wind efficiency $\eta$ versus temperature may therefore be a more valuable tool to understand wind driving for different effective temperature regimes.

\section{Mass-loss rates: expected versus predicted}

Once we have estimated the wind efficiencies from mass-loss rates derived using radio flux densities, we proceeded to compare the results with model predictions. Vink (2000), and Vink et al. (2000a) developed mass-loss rate models and presented them for a $\left(L_{*}, M_{*}\right)$ grid. Based on the models, they produced a recipe to derive a stellar mass-loss rate if the stellar mass, luminosity, effective temperature and terminal velocity are known. We have made use of the routine to derive the expected massloss rates for the radio observed stars. The program also provides information on the position of the star with respect to the BSJ, according to the specific stellar parameters. The predicted mass-loss rates $\dot{M}_{\text {pred }}$ are given in Table 3 .

The recipe to derive the predicted mass-loss rates has helped to discriminate which stars are above and below the BSJ temperature, and we divided them into two groups. Both groups consist of 15 stars. The first group contains: HD 30614, HD 37128 , HD 37742, HD 47432, HD 76341, HD 112244, HD 152424, HD 156154, HD 151804, HD 163181, HD 195592, HD 209975 , HD 204172, BD-11 4586, and Cyg OB2 No. 10. The second group contains the remaining 15 objects.

The errors involved in the derivation of the predicted massloss rates are $\sigma_{L_{*}}=0.5 L_{*}, 0.2 \mathrm{dex}$ in $\log (g)$, and $\sigma_{T \text { eff }}=2000$ $1500 \mathrm{~K}$. A direct comparison between expected and measured values is presented in Fig. 9. We show the wind efficiencies derived using radio observations towards Galactic stars, and,

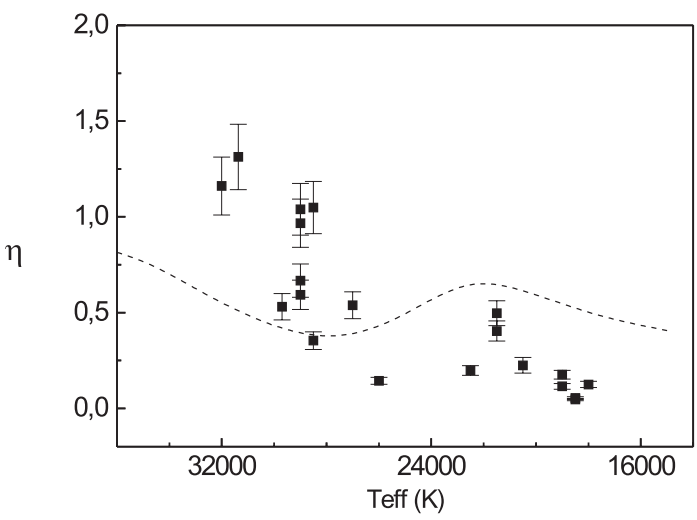

Fig. 9. Modelled wind efficiency for $\log \left(L / L_{\odot}\right)=6.0, M_{*}=60 M_{\odot}$, $v_{\infty} / v_{\text {esc }}=2.6$ (dashed line, same as in Fig. 1) superposed to the wind efficiencies derived by means of radio observations, for Galactic stars (squares).

superposed, the predicted wind efficiency number constructed for $\log \left(L_{*} / L_{\odot}\right)=6.0, M_{*}=60 M_{\odot}, v_{\infty} / v_{\mathrm{esc}}=2.6$, as in Fig. 1 .

Our results show that the stars above the BSJ temperature have predicted mass-loss rates lower than the empirical radio mass-loss rates. The reverse holds true for the stars below the BSJ temperature. If the radio mass-loss rates are affected by significant wind clumping, these empirical rates may need to be reduced by a certain amount, and possibly be brought into agreement with the predictions of radiation-driven wind models. If for stars above the BSJ temperature, the discrepancy between the empirical rates and the theoretical rates is attributable to wind clumping overestimating empirical rates - whilst the theoretical rates are not affected - then, adopting a similar clumping correction for stars below the BSJ temperature, would worsen the discrepancy between the empirical and theoretical rates for $\mathrm{B}$ supergiants.

The reader should be aware that our analysis has been performed using derived mass-loss rates only. "Upper limits" are not taken into account in the comparison between empirical radio rates and mass-loss predictions. Therefore our comparison on the absolute value of the mass-loss rate is necessarily incomplete. Having noted this, there is no a priori reason why incompleteness should be a function of stellar temperature, and although the issue of the absolute mass-loss rates of hot-star winds is still an open one, the qualitative comparison between the empirical radio mass-loss rates and the Monte Carlo predictions of radiation-driven wind models appears to support the predicted mass-loss BSJ. More data around the BSJ will be necessary to confirm this.

\section{Comparison of radio and $\mathrm{H} \alpha$ results}

Many of the stars composing our sample have mass-loss rates in common with measurements from $\mathrm{H} \alpha$ profiles. The values of $\dot{M}_{\mathrm{H} \alpha}$ are listed in Table 3, with their references (Lamers \& Leitherer 1993; Scuderi et al. 1992, 1998; Puls et al. 2006; Crowther et al. 2006; Morel et al. 2004; Herrero et al. 2002).

Additionally, three new $\mathrm{H} \alpha$ mass-loss rates have been derived specifically for this work. The stars HD 42087, HD 148379, and HD 151804 were analyzed in an identical manner to Crowther et al. (2006). For the first two stars the analysis was based on VLT/UVES data (PoP Survey, Bagnulo et al. 2003). For HD 151804, AAT/UCLES spectroscopy was used, and the data was re-analyzed using current version of code plus TLUSTY velocity structure at depth. Figure 10 displays the $\mathrm{H} \alpha$ 


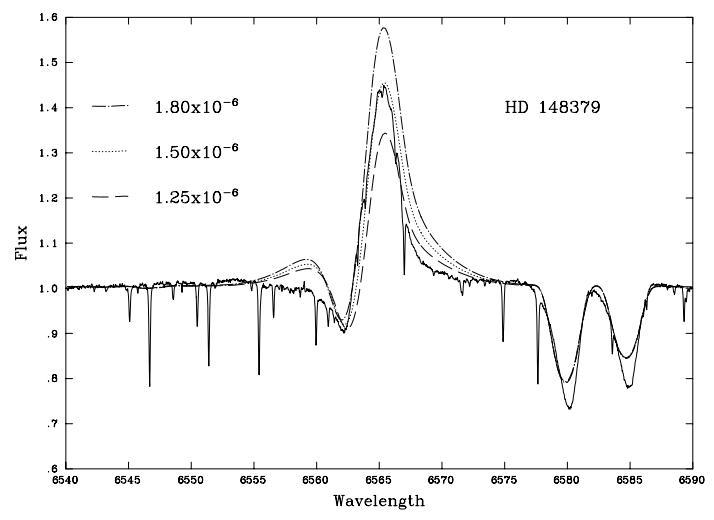

Fig. 10. $\mathrm{H} \alpha$ profile of HD 148379. Source: UVES PoP Survey.

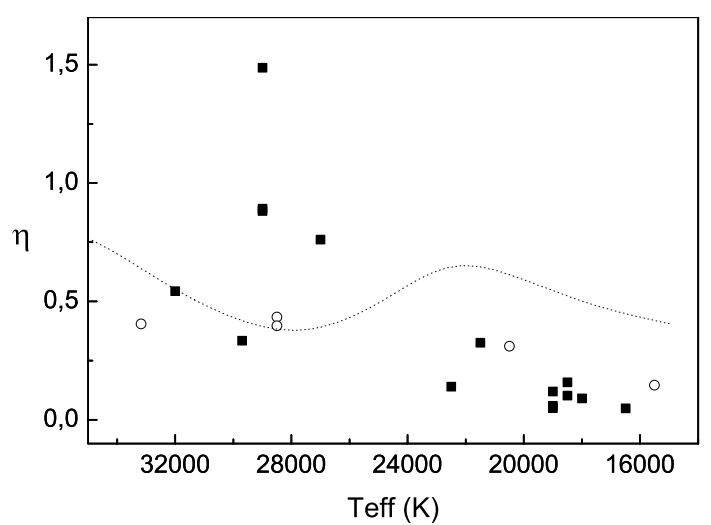

Fig. 11. Wind efficiencies from $\mathrm{H} \alpha$ measurements for the sample stars. Filled squares: results from CMFGEN/FASTWIND profile fitting. Open squares: results from other methods. Dashed line: same as Fig. 1.

profile of HD 148379 with errors, showing the reliability with which mass-loss rates can be achieved.

Figure 11 presents the wind efficiencies derived using massloss rates from $\mathrm{H} \alpha$ profiles for 20 stars out of the present sample (see Table 3). Results from modern CMFGEN and FASTWIND profile fitting are represented with filled squares. The rest (open squares) are specified in Table 3 with brackets. The results with $T_{\text {eff }}$ are more scattered than they were for the radio wind efficiencies, but they do not contradict the predicted behaviour.

An analogous plot to the Galactic $\mathrm{H} \alpha$ one can be constructed for extragalactic stars. Trundle et al. (2004), and Trundle \& Lennon (2005) have obtained the mass-loss rates of 16 B supergiants earlier than B4 in the Small Magellanic Cloud, by means of $\mathrm{H} \alpha$ measurements, all treated in a homogeneous way. These $\dot{M}_{\mathrm{H}_{\alpha}}$ are shown in Fig. 12, as a function of the temperature scale adopted in this work. Possibly, the wind efficiencies for some stars around $22000 \mathrm{~K}$ are above the overall decreasing trend, but larger samples are required to test if the SMC mass-loss rates indeed have a local maximum around the BSJ.

Studies of the comparison between mass-loss rates derived using different methods have been conducted by various groups. Very recently, Fullerton et al. (2006) derived mass-loss rates from UV profiles and investigated whether the UV rates matched those estimated from $\mathrm{H} \alpha$ profiles and radio observations. They found that the results follow the relation: $\dot{M}_{\mathrm{UV}}<\dot{M}_{\mathrm{H} \alpha}<\dot{M}_{\text {radio }}$ and attributed this discrepancy to the presence of wind clumping.

In the present study we have assumed that the free-free radio emission is due to smooth winds. If the winds are significantly clumped, the radio-rates derived in our paper are likely overestimates (see e.g. Blomme et al. 2003). We constructed Fig. 13

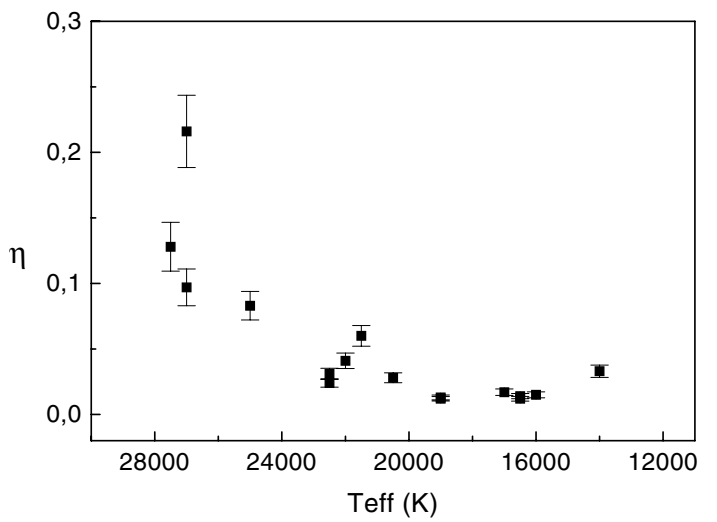

Fig. 12. Wind efficiencies from $\mathrm{H} \alpha$ measurements for SMC stars (from Trundle et al. 2004; and Trundle \& Lennon 2005).

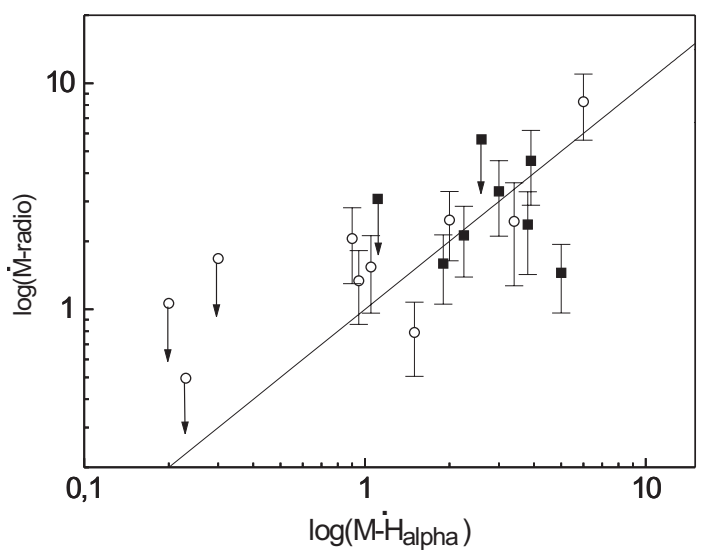

Fig. 13. $\mathrm{H} \alpha$-derived versus radio-derived mass-loss rates. Units are $10^{-6} M \odot \mathrm{yr}^{-1}$. Squares: stars above the BSJ temperature; open circles: stars below the jump temperature. Symbols with arrows represent upper limits in $\dot{M}_{\text {radio }}$.

with all radio and $\mathrm{H} \alpha$ mass-loss rate results available. From the figure it is clear that there is a good match between both sets of data, although the $\mathrm{H} \alpha$ lines are formed in the inner wind, whilst the radio emission arises from the outer wind. This agreement does not necessarily discard clumping, but it may support the hypothesis that the clumping structure is uniformly distributed throughout the whole extension of the wind.

A similar agreement between the inner and the outer wind was found for a large sample of $\mathrm{O}$ stars by Lamers \& Leitherer (1993). However, these results have been challenged by Fullerton et al. (2006) and Puls et al. (2006).

\section{Summary and conclusions}

Throughout the present investigation, a database of radio data of 30 O8-B3 supergiants has been constructed, with observations carried out by different authors. The stellar distances have been reviewed and re-derived, using a subroutine that takes into account the IR and optical indices. We have defined a uniform stellar temperature scale for the spectral range O8-B3.

We have performed high resolution continuum radio observations towards 12 O8-B3 supergiants, detecting three of them: HD 76341, HD 148379 and HD 154090, and derived their massloss rates. The rates of another 18 stars were re-determined using results from the literature.

New $\mathrm{H} \alpha$ mass-loss rates have been obtained for three objects: HD 42087, HD 148379, and HD 151804. The comparison 
of mass-loss rates derived from $\mathrm{H} \alpha$ and radio observations yielded rather good agreement, contrary to some results from previous investigations. However, we note that there are outliers/significant discrepancies for individual cases.

The renormalization of radio observations allowed us to derive a new calibration for the WLR for supergiants of spectral types in the range $\mathrm{O} 8$ to $\mathrm{B} 0$.

The comparison between predicted ( $p$-values) and radioderived ( $r$-values) mass-loss rates yields different results for stars above and below the BSJ temperature. For the latter group we find $p$-values that are larger than the $r$-values, whilst we find the contrary for the first group. The discrepancy in the first group may be attributed to wind clumping, but this would not explain the discrepancy for the objects below the BSJ temperature. An investigation into the effect of wind clumping on the predictions, for objects both above and below the BSJ temperature, would shed light on this issue.

Our radio study of 30 supergiants of spectral types O8-B3 has revealed that for stars around the BSJ, the wind efficiency appear to be above the predicted general declining function towards later spectral types. The temperature of the potential local maximum also agrees with the predicted temperature of the BSJ. Possibly, a similar behaviour is present for the $\mathrm{H} \alpha$ results from SMC stars by Trundle \& Lennon (2005).

From the analysis of the WLR obtained here, we found the corresponding empirical CAK force multiplier to lie in the range $\alpha_{\text {eff }}=0.78-1$. For these large values of $\alpha_{\text {eff }}$, the concept of the WLR may loose its meaning. Studies of the wind efficiency as a function of effective temperature should be considered a powerful alternative to the more often used WLR.

Acknowledgements. We are indebted to the referee, Dr. J. Cassinelli, for critical comments that helped to improve this work, and thank S. Johnston for assistance with observations. P.B. thanks F.A. Bareilles for help in figure handling. P.B. was supported in part by grant BID 1728/OC-AR PICT 03-13291 (ANPCyT) and CONICET (PIP 5375). J.S.V. acknowledges RCUK for his Academic Fellowship and interesting discussions with Drs. D. Lennon and C. Trundle about B supergiants. J.M. acknowledges support by grant AYA2004-07171-C02-02 of Spanish government, FEDER funds, and FQM322 of Junta de Andalucía. This work has made use of the PoP Survey, from the UVES Paranal Observatory Project (ESO DDT Program ID 266.D-5655).

\section{References}

Abbott, D. C., Bieging, J. H., Churchwell, E., \& Cassinelli, J. P. 1980, ApJ, 238, 196

Bagnulo, S., Jehin, E., Ledoux, C., et al. 2003, Messenger, 114, 10

Benaglia, P., Cappa, C. E., \& Koribalski, B. 2001, A\&A, 200, 58

Bieging, J. H., Abbott, D. C., \& Churchwell, E. B. 1989, ApJ, 340, 518

Blomme, R., van de Steene, G. C., Prinja, R. K., et al. 2003, A\&A, 408, 715

Bohannan, B., \& Crowther, P. A. 1999, ApJ, 511, 374

Bouret, J.,-C., Lanz, T., \& Hillier, D. J. 2003, ApJ, 595, 1182

Castor, J. I., Abbott, D. C., \& Klein, R. I. 1975, ApJ, 195, 157

Chiosi, C., \& Maeder, A. 1986, ARA\&A, 24, 329

Crawford, D. L., Barnes J. V., \& Golson, J. C. 1971, AJ, 76, 1058

Crowther, P. A., \& Bohannan, B. 1997, A\&A, 317, 532

Crowther, P. A., Lennon, D. J., \& Walborn, N. R. 2006, A\&A, 446, 279

Curé, M., Rial, D. F., \& Cidale, L. 2005, A\&A, 437, 929

Davies, B., Vink, J. S., \& Oudmaijer, R. D. 2007, A\&A, to be submitted Eldridge, J. J., \& Vink, J. S. 2006, A\&A, 452, 295

Evans, C. J., Lennon, D. J., Walborn, N. R., et al. 2004, PASP, 116, 909

Eversberg, T., Lépine, S., \& Moffat, A. F. J. 1998, ApJ, 494, 799
Feinstein, A., \& Marraco, H. G. 1979, AJ, 84, 1713

Fullerton, A. W., Massa, D. L., \& Prinja, R. K. 2006, ApJ, 637, 1025

Garrison, R. F., Hiltner, W. A., \& Schild, R. E. 1977, ApJS, 35, 111

Herrero, A., Puls, J., \& Najarro, F. 2002, A\&A, 396, 949

Heger, A., Fryer, C. L., Woosley, S. E., et al. 2003, ApJ, 591, 288

Hillier, D. J., \& Miller, D. L. 1998, ApJ, 496, 407

Hiltner, W. A., Garrison, R. F., \& Schild, R. E. 1969, ApJ, 1157, 313

Howarth, I. D., Siebert, K. W., Hussain, G. A. J., \& Prinja, R. K. 1997, MNRAS, 284,265

Humpreys, R. M. 1978, ApJS, 38, 309

Hunter, I., Smoker, J. V., Keenan, F. P., et al. 2006, MNRAS, 367, 1478

Johnson, H. L., Mitchell, R. I., Iriarte, B., \& Wisniewski, W. Z. 1966, Comm. Lunar Plan. Lab. IV, No 63

Kotak, R., \& Vink, J. S. 2006, A\&A, 460, L5

Kozok, J. R. 1985, A\&AS, 61, 387

Kudritzki, R., \& Puls, J. 2000, ARA\&A, 629

Lamers, H. J. G. L. M., \& Pauldrach, A. W. A. 1991, A\&A, 244, L5

Lamers, H. J. G. L. M., \& Leitherer, C. 1993, ApJ, 412, 771

Lamers, H. J. G. L. M., Snow, T. P., \& Lindholm, D. M. 1995, ApJ, 455, 269

Leitherer, C., Chapman, J. M., \& Koribalski, B. 1995, ApJ, 450, 289

Limongi, M., \& Chieffi, A. 2006, ApJ, 647, 483

Lesh, J. R. 1968, ApJ, 517, 371

Lucy, L. B., \& Solomon, P. M. 1970, ApJ, 159, 879

Maeder, A., \& Meynet, G. 2003, A\&A, 411, 543

Maíz-Apellániz, J., Walborn, N. R., Galué, H. A., \& Wei, L. H. 2004, ApJS, 151, 103

Maíz Apellániz, J. 2004, PASP, 116, 859

Markova, N., Puls, J., Repolust, T., \& Markov, H. 2004, A\&A, 413, 693

Martins, F., Schaerer, D., \& Hillier, D. J. 2005, A\&A, 436, 1049

Massey, P., \& Thompson, A. B. 1991, AJ, 101, 1408

Moffett, A. T., \& Barnes, T. G. 1979, PASP, 91, 289

Mokiem, M. R., de Koter, A., Vink, J. S., et al. 2007, A\&A, submitted

Morel, T., Marchenko, S. V., Pati, A. K., et al. 2004, MNRAS, 351, 552

Morgan, W. W., Code, A. D., \& Whitford, A. E. 1955, ApJS, 2, 41

Panagia, N., \& Felli, M. 1975, A\&A, 39, 1

Pauldrach, A. W. A., \& Puls, J. 1990, A\&A, 237, 409

Pelupessy, I., Lamers, H. J. G. L. M., \& Vink, J. S. 2000, A\&A, 359, 695

Perryman, M. A. C., Lindegren, L., Kovalevsky, J., et al. 1997, A\&A, 323, L49

Prinja, R. K., Barlow, K. J., \& Howarth, I. D. 1990, ApJ, 361, 607

Prinja, R. K., Massa, D., \& Searle, S. C. 2005, A\&A, 403, L41

Puls, J., Markova, N., Scuderi, S., et al. 2006, A\&A, 454, 652

Rauw, G., Nazé, Y., Carrier, F., et al. 2001, A\&A, 366, 585

Repolust, T., Puls, J., \& Herrero, A. 2004, A\&A, 415, 349

Schild, R. E., Garrison, R. F., \& Hiltner W. A. 1983, ApJS, 51, 321

Scuderi, S., Bonanno, G., di Benedetto, R., et al. 1992, ApJ, 392, 201

Scuderi, S., Panagia, N., Stanghellini, et al. 1998, A\&A, 332, 251

Setia Gunawan, D. Y. A., de Bruyn, A. G., van der Hucht, K. A., \& Williams, P. M. 2000, A\&A, 356, 676

Smith, N., Vink, J. S., \& de Koter, A. 2004, ApJ, 615, 475

Thaller, M. L., Gies, D. R., Fullerton, A. W., et al. 2001, ApJ, 554, 1070

Trundle, C., Lennon, D. J., Puls, J., \& Dufton, P. L. 2004, A\&A, 417, 217

Trundle, C., \& Lennon, D. J. 2005, A\&A, 434, 677

Vink, J. S. 2000, Ph.D. Thesis, University of Utrecht

Vink, J. S. 2006, in Stellar Evolution at Low Metallicity: Mass Loss, Explosions, Cosmology, ed. H. J. G. L. M. Lamers, N. Langer, T. Nugis, \& K. Annuk, PASP Conf. Ser., 353, 113

Vink, J. S., \& de Koter, A. 2002, A\&A, 393, 543

Vink, J. S., de Koter, A., \& Lamers, H. J. G. L. M. 1999, A\&A, 350, 181

Vink, J. S., de Koter, A., \& Lamers, H. J. G. L. M. 2000a, A\&A, 362, 295

Vink, J. S., de Koter, A., \& Lamers, H. J. G. L. M. 2000b, ASPC, 204, 427

Vink, J. S., de Koter, A., \& Lamers, H. J. G. L. M. 2001, A\&A, 369, 574

Walborn, N. R. 1972a, AJ, 77, 312

Walborn, N. R. 1972b, ApJ, 176, 119

Walborn, N. R. 1973, AJ, 78, 1067

Walborn, N. R. 1976, ApJ, 205, 419

Walborn, N. R. 1977, ApJ, 176, 116

Walborn, N. R. 1982, AJ, 87, 1300

Williams, P. M. 1996, ASP Conf. Ser., 93, 15

Wright, A. E., \& Barlow, M. J. 1975, MNRAS, 170, 41 